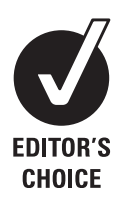

CHOICE

\begin{abstract}
${ }^{1}$ MRC Clinical Sciences Centre, Imperial College, London, UK ${ }^{2}$ Department of Imaging, Imperial College Healthcare NHS Trust, London, UK ${ }^{3}$ National Heart and Lung Institute, Imperial College, London, UK
\end{abstract}

\section{Correspondence to} Dr Declan P O'Regan, MRC Clinical Sciences Centre, Robert Steiner MRI Unit, Faculty of Medicine, Imperial College, Hammersmith Hospital Campus, Du Cane Road, London W12 ONN, UK;

declan.oregan@csc.mrc.ac.uk

Accepted 15 September 2010 Published Online First 21 October 2010

\title{
Assessment of severe reperfusion injury with T2* cardiac MRI in patients with acute myocardial infarction
}

\author{
Declan P O'Regan, ${ }^{1,2}$ Ben Ariff, ${ }^{1,2}$ Clare Neuwirth, ${ }^{1}$ Yvonne Tan, ${ }^{1}$ Giuliana Durighel, ${ }^{1}$ \\ Stuart A Cook ${ }^{1,3}$
}

\section{ABSTRACT}

Background In patients with acute myocardial infarction, restoration of coronary flow by primary coronary intervention (PCI) can lead to profound ischaemiareperfusion injury with detrimental effects on myocardial salvage. Non-invasive assessment of interstitial myocardial haemorrhage by $\mathrm{T} 2^{*}$ cardiac MRI (T2*-CMR) provides a novel and specific biomarker of severe reperfusion injury which may be of prognostic value.

Objective To characterise the determinants of acute ischaemia-reperfusion injury following ST elevation myocardial infarction (STEMI) using CMR.

Methods and results Fifty patients with acute STEMI who had been successfully treated by $\mathrm{PCI}$ were studied. T2* -CMR was used to identify the presence of reperfusion haemorrhage and contrast enhancement was used to measure microvascular obstruction (MVO) and infarct size. Haemorrhagic ischaemia-reperfusion injury was present in 29 patients (58\%) following $\mathrm{PCl}$ and occurred despite rapid revascularisation (mean $4.2 \pm 3.3 \mathrm{~h}$ ). Haemorrhage was only present when the infarct involved at least $80 \%$ (mean \pm SD $91 \pm 5.3 \%$ ) of the left ventricular wall thickness. There was a strong association between the extent of MVO and reperfusion haemorrhage $\left(r^{2}=0.87, p<0.001\right)$. Transmural infarcts $(n=43)$ showed significantly impaired systolic wall thickening at the infarct mid point when reperfusion haemorrhage was present $(21.5 \pm 16.7 \%$ vs $3.7 \pm 12.9 \%), p<0.0001)$ compared with nonhaemorrhagic infarcts.

Conclusions Severe reperfusion injury may occur when there is near-transmural myocardial necrosis despite early and successful revascularisation. Reperfusion haemorrhage is closely associated with the development of MVO. These findings indicate that, once advanced necrosis has developed, the potential for severe myocardial reperfusion injury is significantly enhanced.

Early restoration of myocardial perfusion is the most important goal of treating patients with acute ST elevation myocardial infarction (STEMI). ${ }^{1}$ However, despite re-establishing patency of the infarct-related coronary artery, achieving optimal tissue perfusion and myocyte salvage in all patients has remained elusive. ${ }^{2}$ The process of restoring blood flow to ischaemic myocardium can induce damage itself, and this phenomenon-termed ischaemia-reperfusion injury-can paradoxically reduce the beneficial effects of myocardial reperfusion. ${ }^{3}$ This process results in the death of cardiac myocytes that were viable immediately prior to myocardial reperfusion and may be responsible for up to half the final infarct size. ${ }^{4}$

Determining the conditions under which reperfusion injury occurs in clinical practice would provide a rationale for treating or avoiding this therapeutic complication. In recent years there has been increasing interest in cardiac MRI for assessing myocardial ischaemic injury. ${ }^{5} \mathrm{~T} 2$-weighted imaging of myocardial oedema has been shown to be an accurate method of retrospectively determining the extent of ischaemic tissue in both animal models and in clinical practice after primary coronary intervention (PCI). ${ }^{6} 7$ Following revacularisation, myocardial oedema is present in both non-reperfused $^{8}$ and reperfused infarcts ${ }^{7}$; however interstitial myocardial haemorrhage is only observed following coronary reperfusion and serves as a specific marker of severe ischaemia-reperfusion injury. ${ }^{9-11}$ Furthermore, the presence of interstitial haemorrhage is strongly associated with microvascular obstruction (MVO) which is known to rapidly worsen following reperfusion. ${ }^{12}$

Haemorrhage contains paramagnetic blood degradation products which have characteristic effects on the magnetic field at the microscopic level and can be readily identified with $\mathrm{T} 2^{*}$-weighted cardiac MRI (T2*-CMR). ${ }^{13}$ Mapping the distribution of these blood products provides the only noninvasive method for identifying infarctions where severe reperfusion injury has occurred. We used this approach to assess the conditions which predispose to the development of severe reperfusion injury in patients with acute STEMI treated with PCI.

\section{METHODS}

\section{Patients}

The study was approved by the hospital research ethics committee and all patients gave written informed consent. Fifty patients (48 men, 2 women; age range 29-74 years; mean age 54.8 years) who had undergone PCI within the previous 7 days were prospectively investigated. Fifteen of these subjects had participated in previously published work. ${ }^{13} 14$ The inclusion criteria were men and women aged $18-85$ years with an ECG diagnosis of acute STEMI who were treated with PCI. Exclusion criteria were contraindications to $\mathrm{CMR}$, previous myocardial infarction or heart failure, clinical instability, significant arrhythmias, pregnancy or lactation. The diagnosis of STEMI required ST elevation of $\geq 1 \mathrm{~mm}$ in two contiguous ECG leads and chest pain onset within $12 \mathrm{~h}$ of presentation. 


\section{Coronary intervention}

Prior to PCI the patients received aspirin and clopidogrel, and intravenous heparin was administered as a bolus with the aim of achieving an activated clotting time of $>250$ s. Patients underwent left heart catheterisation using either transfemoral or transradial approaches. Selective coronary catheterisation was used to identify and treat the infarct-related artery and all patients received either a bare metal or drug-eluting stent. Non-culprit atherosclerotic vessels were not treated. In total, 44 patients $(88 \%)$ received a glycoprotein IIb/IIIa inhibitor periprocedurally.

\section{Angiographic analysis}

Flow in the infarct-related artery was graded using the Thrombolysis in Myocardial Infarction trial (TIMI) criteria prior to and after the intervention.

\section{Image acquisition}

The CMR studies were performed on a 1.5T Philips Achieva system (Best, The Netherlands). The maximum gradient strength was $33 \mathrm{mT} / \mathrm{m}$ and the maximum slew rate was $160 \mathrm{mT} / \mathrm{m} / \mathrm{ms}$. A five-element cardiac phased-array coil was used for signal reception.

Scout images were obtained and used to plan an axial stack of cine balanced-steady state free precession (b-SSFP) images in the left ventricular short axis plane from base to apex using the following parameters: voxel size $2.0 \times 2.2 \times 8 \mathrm{~mm}$, flip angle $60^{\circ}$, slice thickness $8 \mathrm{~mm}$ with a $2 \mathrm{~mm}$ gap, TE $1.5 \mathrm{~ms}$, TR $3.0 \mathrm{~ms}$ and 20 cardiac phases. Myocardial oedema was imaged with a navigator-gated black blood T2-weighted turbo spin echo sequence with spectrally selective inversion recovery (SPIR) fat suppression using the following parameters: voxel size $1.9 \times 2.6$ $\times 10 \mathrm{~mm}$, flip angle $90^{\circ}$, slice thickness $10 \mathrm{~mm}$, TE $100 \mathrm{~ms}$, TR 2 $\mathrm{R}-\mathrm{R}$ intervals. Myocardial haemorrhage was imaged with a navigator-gated black blood gradient multiecho $\mathrm{T} 2 *$ sequence using the following parameters: voxel size $1.7 \times 2.8 \times 10 \mathrm{~mm}$, flip angle $20^{\circ}$, slice thickness $10 \mathrm{~mm}$, TR $17 \mathrm{~ms}, 7$ echoes, TE 2.3-16.1 ms, $\Delta$ TE $2.3 \mathrm{~ms}$. A single short-axis image plane was chosen at the level of maximal oedema.

An intravenous bolus of gadolinium dimeglumine (Magnevist; Bayer Schering Pharma, Berlin, Germany) was administered at a dose of $0.2 \mathrm{mmol} / \mathrm{kg}$ via a power injector (Spectris Solaris; Medrad, Indianola, Pennsylvania, USA). Early enhancement imaging was performed with a single breath hold 3D inversion recovery sequence within 1 min of contrast injection using the following parameters: voxel size $1.4 \times 2.1 \times 8 \mathrm{~mm}$, flip angle $15^{\circ}$, slice thickness $8 \mathrm{~mm}$, TE $1.4 \mathrm{~ms}$, TR $4.3 \mathrm{~ms}$. Late enhancement imaging was performed after a 10 min delay with a $2 \mathrm{D}$ inversion recovery sequence using the following parameters: $1.5 \times 1.7 \times 8 \mathrm{~mm}$, flip angle $15^{\circ}$, slice thickness $8 \mathrm{~mm}$, TE $1.4 \mathrm{~ms}$, TR $4.3 \mathrm{~ms}$. The inversion time was adjusted to null the signal from normal myocardium using the Look-Locker method.

\section{Image analysis}

Segmentation of the left ventricular cavity and wall from the short axis b-SSFP cine images was performed using CMRtools (Cardiovascular Imaging Solutions, London, UK). Measurements were made by two experienced observers and the mean value obtained. Left ventricular mass, end diastolic volume and end systolic volume were calculated and indexed to body surface area. End diastolic wall thickness and fractional wall thickening were measured at the mid point of the infarction. The regions of late enhancement and MVO were quantified using an automated algorithm (Segment 1.8, Medviso, Sweden) with a section-specific signal intensity threshold and level-set boundary detection to identify the interfaces between different regions of signal intensity. ${ }^{15} \mathrm{~A}$ single section in the same plane as the short axis $\mathrm{T}^{*}$ image was used for comparative analysis and infarct transmurality was measured along 40 radial cords.

A pixel-by-pixel analysis was performed of the multiecho T2*weighted images using Matlab 2008b (Mathworks, Natick, Massachusetts, USA) with fitting to a simple monoexponential decay. The T2* decay constant was measured in both haemorrhagic and non-haemorrhagic myocardium. The proportion of pixels within infarcted myocardium with a T2* value of $<20 \mathrm{~ms}$ (retrospectively determined as 2SD below the mean of remote myocardium in all subjects) was measured and expressed as a percentage of the left ventricular area of that section. Reperfusion haemorrhage was considered to be present if the area of abnormal T2* within the confines of the infarct constituted at least $1 \%$ of the left ventricular area in that section. Susceptibility artefact adjacent to the inferolateral left ventricular epicardium was manually excluded if present. Haemorrhage was considered to be present on T2-weighted imaging if there was a hypointense area within the area at risk with a mean signal intensity 2SD below the signal intensity of myocardial oedema constituting at least $1 \%$ of the left ventricular area in that section. ${ }^{16}$ The proportion of area at risk on T2-weighted imaging which did not show late enhancement was used to calculate the myocardial salvage index.

\section{Statistical analysis}

All continuous data are reported as mean \pm 1 SD. Comparison of continuous variables between groups was performed with the Mann-Whitney U test. Categorical variables were compared with the $\chi^{2}$ test. The association between variables was assessed by linear least squares regression. In each case a $p$ value of $<0.05$ was considered statistically significant. Univariate and multivariate regression analysis was used to assess the predictive value of CMR findings for post-PCI ejection fraction. Variables were retained in the model if they contributed to the explanatory power of the regression equation with a $p$ value $<0.10$.

\section{RESULTS}

\section{Patient characteristics}

On the basis of T2*-CMR, 29 patients had haemorrhagic infarctions and 21 patients had non-haemorrhagic infarctions. The baseline parameters are shown in table 1. For all patients the mean time from symptom onset to reperfusion was $3.9 \pm 2.8 \mathrm{~h}$ (range 1-12). The baseline CMR was performed at a mean of $3.1 \pm 2.0$ days (range $1-7$ ) after PCI. All subjects demonstrated myocardial oedema within the vascular territory of the culprit artery and this was invariably associated with a regional wall motion abnormality. On late enhancement images all patients showed a single contiguous zone of myocardial necrosis. Late enhancement was located within the zone of oedema in each case. The mean infarct size for all patients was $18.9 \pm 9.3 \%$ (range $6-41 \%$ ) with a mean salvage index of $45.2 \pm 21.6 \%$ (range $0-85 \%)$.

\section{Microvascular obstruction and reperfusion injury}

MVO was present in 29 patients (58\%). The mean area of early MVO was $19.0 \pm 11.0 \%$ (range $5.4-40.5 \%$ ) and late MVO was $6.6 \pm 4.8 \%$ (range $1.0-18.5 \%$ ). Small regions of MVO were typically confined to the subendocardium but, in more extensive infarcts, only the subepicardium was spared. Focal myocardial haemorrhage was identified with $\mathrm{T}^{*}$ imaging in all patients 
Table 1 Baseline characteristics of patients undergoing primary coronary intervention

\begin{tabular}{|c|c|c|c|}
\hline Variable & $\begin{array}{l}\text { Haemorrhagic } \\
\text { STEMI }(n=29)\end{array}$ & $\begin{array}{l}\text { Non-haemorrhagic } \\
\text { STEMI }(\mathrm{n}=21)\end{array}$ & p Value \\
\hline Age (years) & $55 \pm 11$ & $54 \pm 11$ & NS \\
\hline $\mathrm{M} / \mathrm{F}$ & $28 / 1$ & $20 / 1$ & NS \\
\hline Body surface area $\left(\mathrm{m}^{2}\right)$ & $1.96 \pm 0.18$ & $1.99 \pm 0.17$ & NS \\
\hline Systolic blood pressure (mm Hg) & $132 \pm 33$ & $125 \pm 20$ & NS \\
\hline Diastolic blood pressure (mm $\mathrm{Hg}$ ) & $84 \pm 20$ & $76 \pm 15$ & NS \\
\hline Heart rate $(\mathrm{bpm})$ & $74 \pm 16$ & $79 \pm 19$ & NS \\
\hline Diabetes mellitus & $6(21 \%)$ & $1(5 \%)$ & NS \\
\hline Current or ex-smoker & $14(48 \%)$ & $15(72 \%)$ & NS \\
\hline Hypertension & $8(28 \%)$ & $6(29 \%)$ & NS \\
\hline Time from symptoms to reperfusion (h) & $4.2 \pm 3.3$ & $3.7 \pm 2.3$ & NS \\
\hline Peak creatinine kinase (IU/I) & $3235 \pm 1938$ & $1683 \pm 1604$ & $<0.01$ \\
\hline Peak troponin I $(\mu \mathrm{g} / \mathrm{l})$ & $111 \pm 103$ & $49 \pm 59$ & $<0.05$ \\
\hline \multicolumn{4}{|l|}{ Culprit coronary artery } \\
\hline Left anterior descending & $19(66 \%)$ & $7(33 \%)$ & $<0.05$ \\
\hline Circumflex artery & $2(7 \%)$ & $4(19 \%)$ & NS \\
\hline Right coronary & $8(28 \%)$ & $10(48 \%)$ & NS \\
\hline \multicolumn{4}{|l|}{ TIMI flow pre-PCI } \\
\hline Grade 0 & $27(93 \%)$ & $16(76 \%)$ & NS \\
\hline Grade I & $2(7 \%)$ & $1(5 \%)$ & NS \\
\hline Grade II & 0 & $2(10 \%)$ & NS \\
\hline Grade III & 0 & $2(10 \%)$ & NS \\
\hline \multicolumn{4}{|l|}{ TIMI flow post-PCI } \\
\hline Grade II & $2(7 \%)$ & 0 & NS \\
\hline Grade III & $27(93 \%)$ & $21(100 \%)$ & NS \\
\hline \multicolumn{4}{|l|}{ Medication given during admission } \\
\hline Aspirin & $29(100 \%)$ & $21(100 \%)$ & NS \\
\hline Clopidogrel & $28(97 \%)$ & $20(95 \%)$ & NS \\
\hline Beta-blocker & $25(86 \%)$ & $18(86 \%)$ & NS \\
\hline $\mathrm{Ca}^{2+}$ channel blocker & $1(3 \%)$ & $1(5 \%)$ & NS \\
\hline ACE inhibitor & $29(100 \%)$ & $18(86 \%)$ & NS \\
\hline Statin & $6(21 \%)$ & $4(19 \%)$ & NS \\
\hline Glycoprotein Ilb/Illa antagonist & $27(93 \%)$ & $17(81 \%)$ & NS \\
\hline Heparin & $29(100 \%)$ & $21(100 \%)$ & NS \\
\hline
\end{tabular}

with MVO and the regions of haemorrhage and MVO were colocalised (figure 1). The mean area of haemorrhage was $7.7 \pm 6.2 \%$ (range 1.0-25.4\%) and was present in infarcts with a cross-sectional area between $17 \%$ and $57 \%$ of the left ventricle. The area of haemorrhage showed a strong correlation to the corresponding area of late MVO $\left(\mathrm{r}^{2}=0.87, \mathrm{p}<0.001\right)$ but a weaker correlation with the larger and more variable area of early MVO $\left(r^{2}=0.30, p<0.003\right)$.

\section{T2-weighted imaging versus T2* mapping}

T2* mapping had a sensitivity of $100 \%$ for detecting the presence of MVO in an equivalent section. Susceptibility artefact, particularly for lateral wall infarcts, resulted in a specificity of $90 \%$. The mean $\mathrm{T}^{*}$ within haemorrhagic infarctions was $15.4 \pm 5.7 \mathrm{~ms}$ compared with $47.2 \pm 13.8 \mathrm{~ms}$ in non-haemorrhagic myocardium. T2-weighted imaging of oedema had a sensitivity of $82 \%$ for detecting $\mathrm{MVO}$, and an example of a false negative study is shown in figure 2. The specificity for T2-weighted imaging was also $90 \%$.

\section{Characteristics of haemorrhagic infarctions}

Haemorrhagic infarctions were observed in each of the main coronary artery territories. In haemorrhagic infarcts, all 29 patients had either TIMI grade 0 or I prior to intervention. Following PCI, 27 patients (93\%) had grade III flow and
2 patients (7\%) had grade II flow. All patients were treated with aspirin and heparin periprocedurally, and there was no significant difference in the rates of glycoprotein IIb/IIIa antagonist usage between haemorrhagic and non-haemorrhagic infarcts. Cardiac enzymes (troponin I and creatinine kinase) were significantly greater in the haemorrhagic infarction group $(p<0.05)$. No significant difference in pain-to-balloon time was identified between haemorrhagic and non-haemorrhagic infarcts, with reperfusion haemorrhage detected in subjects who received PCI within the first few hours after the onset of chest pain.

The imaging findings are summarised in table 2. Infarct volume was significantly greater when haemorrhage was present $(23.8 \pm 8.2 \%$ vs $12.0 \pm 5.9 \%, p<0.0001)$. The left ventricular ejection fraction (LVEF) was lower in the haemorrhage group $(51 \pm 11 \%$ vs $59 \pm 7 \%, \mathrm{p}<0.01)$. Using univariate analysis, the area of reperfusion haemorrhage showed a strong association with LVEF $(\beta=-0.56, p<0.0001)$. After multivariate analysis, infarct volume remained the only independent predictor of LVEF $(\beta=-0.60, p<0.01)$.

Excluding the seven patients with partial thickness infarcts (necrosis $<80 \%$ of left ventricular wall), fractional wall thickening at the infarct mid point showed significantly greater impairment when reperfusion haemorrhage was present $(21.5 \pm 16.7 \%$ vs $3.7 \pm 12.9 \%, p<0.001)$. Haemorrhagic infarction was associated with significantly greater end diastolic volume 
Figure 1 Cardiac MRI of acute reperfusion injury. Images from three patients (A, B and C) with acute ST elevation myocardial infarction obtained within $48 \mathrm{~h}$ of primary coronary intervention. The left panels show the $\mathrm{T}^{*}$ maps with areas of haemorrhage depicted in red. The right panels show the delayed contrast-enhanced images. (A) A left anterior descending coronary artery occlusion showing a close correspondence between reperfusion haemorrhage and microvascular obstruction (MVO) (arrows) in the anterior wall. (B) A right coronary artery occlusion demonstrating subendocardial haemorrhage and MVO in the inferior wall (arrow). (C) An obtuse marginal artery occlusion resulting in a partial thickness infarct of the lateral wall (arrow) and posterior papillary muscle; however, neither haemorrhage nor MVO is present in this case.
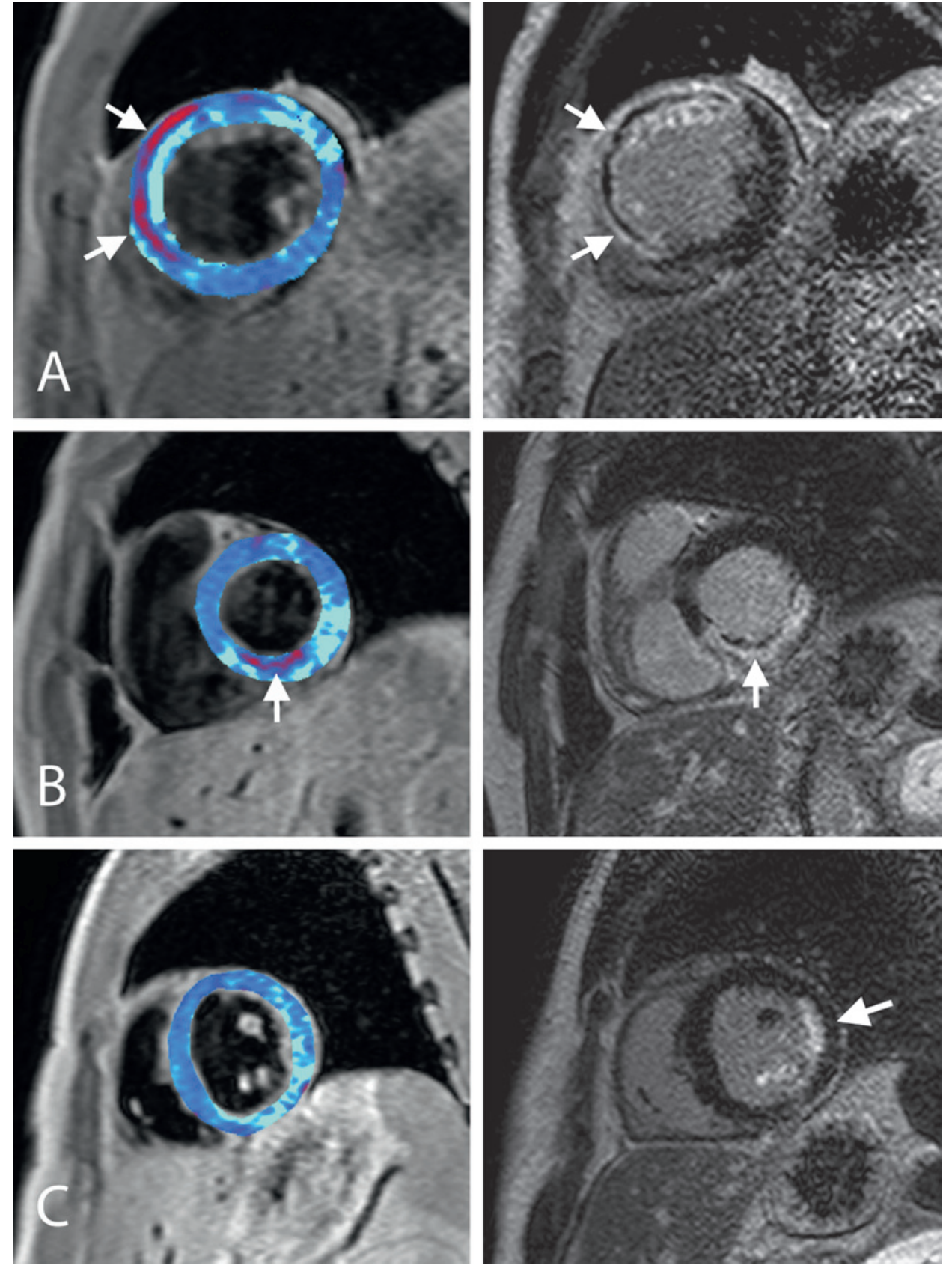

index $\left(81.0 \pm 21.4 \mathrm{ml} / \mathrm{m}^{2}\right.$ vs $\left.67.1 \pm 11.4 \mathrm{ml} / \mathrm{m}^{2}, \mathrm{p}<0.001\right)$ and end systolic volume index $\left(41.1 \pm 17.1 \mathrm{ml} / \mathrm{m}^{2}\right.$ vs $28.3 \pm 7.9 \mathrm{ml} / \mathrm{m}^{2}$, $\mathrm{p}<0.001)$. No significant difference was found between end diastolic wall thickness at the infarct mid point, although left ventricular mass was elevated in haemorrhagic infarctions $\left(84 \pm 16 \mathrm{~g} / \mathrm{m}^{2}\right.$ vs $\left.73 \pm 11 \mathrm{~g} / \mathrm{m}^{2}, \mathrm{p}<0.01\right)$. The relationship between cross-sectional area of the infarct and infarct transmural thickness for both haemorrhagic and non-haemorrhagic infarcts is shown in figure 3. A plot of the relative extent of myocardial oedema, infarct, early $\mathrm{MVO}$, late $\mathrm{MVO}$ and haemorrhage is shown in figure 4 .

\section{DISCUSSION}

Ischaemia-reperfusion injury is known to have a major influence on myocyte salvage and final infarct size. Our study has used a novel imaging technique to quantify the presence of myocardial haemorrhage which is indicative of severe reperfusion injury in post-STEMI patients. We observed that reperfusion injury is rarely present in smaller subendocardial infarcts but is a frequent complication in large infarcts where necrosis is almost transmural, which can occur even in rapidly reperfused infarcts. These findings indicate that, as necrosis becomes established throughout the ischaemic bed, the potential for an adverse response to reperfusion is significantly enhanced.

The concept of lethal reperfusion injury as an independent mediator of myocyte death, as distinct from an exacerbation of ischaemic injury, is controversial. ${ }^{3}$ The difficulty lies in determining the progress of necrosis during the transition from ischaemia to reperfusion. ${ }^{4}$ Although T2-weighted imaging allows determination of the ischaemic area at risk, it is not possible to retrospectively determine the effect of reperfusion on oedema and necrosis as both of these features are also observed in non-reperfused infarcts. While ischaemia-reperfusion injury has an effect on vulnerable myocytes throughout the vascular 

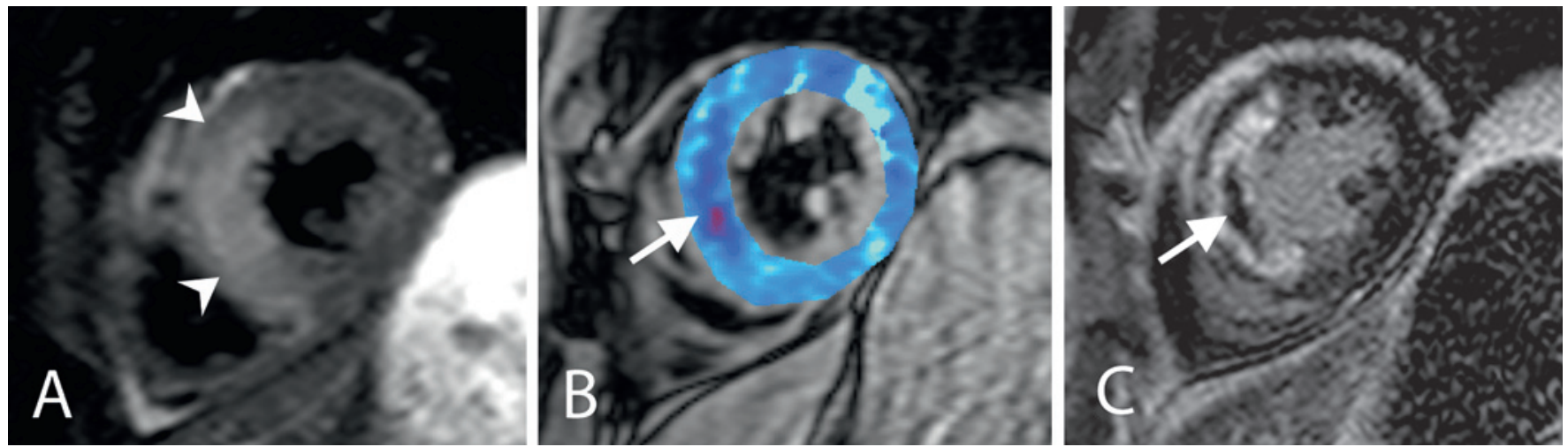

Figure 2 Reperfusion haemorrhage only apparent on T2* imaging. Cardiac MRI images obtained from a patient 3 days after primary coronary intervention to a left anterior descending coronary artery. (A) T2-SPIR (spectrally selective inversion recovery) imaging shows uniform acute myocardial oedema in the septum (arrowheads). (B) A T2* map shows the areas of reperfusion haemorrhage in red (arrow). This closely corresponds to the region of persistent microvascular obstruction on the late enhancement image (C, arrow) which is surrounded by enhancing necrosis.

bed of the culprit artery, ${ }^{17}$ the development of haemorrhage appears to be a specific finding that occurs only after flow has been restored to severely ischaemic myocardium. ${ }^{9-11}$ The technique of $\mathrm{T}^{*}$ imaging used in our study has been validated in both preclinical and ex vivo studies as an effective method for quantifying reperfusion haemorrhage and comparing its distribution with other measures of ischaemic damage. ${ }^{18} 19$

In our study, reperfusion injury was not restricted to late presentations and in some cases haemorrhage was present despite early and successful coronary intervention. We observed severe reperfusion injury in infarcts with poor myocardial salvage, especially in the left anterior descending (LAD) coronary artery territory, and did not find a strong association with painto-balloon time. This may reflect the wide interindividual variation in salvage rates achieved with $\mathrm{PCI} .{ }^{20}$ Indeed, our findings indicate that, once necrosis has become established within the ischaemic bed, reperfusion haemorrhage may occur rapidly and extensively within the infarct. We also observed that haemorrhage occurs in the subendocardium and mid wall, which is consistent with the secondary wavefront of reperfusion injury seen in animals. ${ }^{21}$ We found that reperfusion haemorrhage consistently coincided with the area of irreversible microcirculatory damage (MVO). The extent of MVO increases significantly even after the restoration of coronary flow. ${ }^{12}$ The mechanism for this is unknown, although vascular microplugging by platelets and neutrophils, myocyte cell swelling and free radical-mediated endothelial injury have all been proposed. ${ }^{22}$
In animal studies the advance of reperfusion haemorrhage lags behind the expansion of $\mathrm{MVO}^{21}$ but the relationship between these phenomena in clinical practice is unclear, especially as patients are routinely heparinised and treated with antiplatelet drugs. It has been proposed that, if the expansion of MVO can be closely related to progressive haemorrhage within the myocardium, it might be considered a reperfusion injury which could be potentially avoided or treated. ${ }^{23}$ In our study the highly consistent association of reperfusion haemorrhage with MVO throughout the first 7 days following PCI indicates that haemorrhage expansion has a potential role in the evolution of MVO.

The presence of $\mathrm{MVO}$ is associated with adverse left ventricular remodelling even after controlling for infarct size. ${ }^{24}$ If reperfusion injury is a contributory factor in the development of $\mathrm{MVO}$, it raises the question of whether attempting reperfusion of a completed infarct may worsen the patient's prognosis. Although experimental studies have shown a reduction in infarct expansion and left ventricular dilation even when late reperfusion has been initiated in completed infarcts, ${ }^{25} 26$ clinical trials have failed to show that the 'open-artery hypothesis' leads to improved clinical outcomes. ${ }^{27} 28$ Studies of the prognostic significance of reperfusion haemorrhage as an independent predictor of remodelling have been inconclusive although, until recently, investigators have relied on detecting signal voids on T2-weighted imaging within the area of myocardial oedema to infer the presence of haemorrhage. ${ }^{16}{ }^{29}$ In the study of reperfusion injury, $\mathrm{T} 2 *-\mathrm{CMR}$ is advantageous as it is more sensitive to

Table 2 Cardiac MRI parameters following primary coronary intervention

\begin{tabular}{|c|c|c|c|}
\hline Variable & $\begin{array}{l}\text { Haemorrhagic STEMI } \\
(n=29)\end{array}$ & $\begin{array}{l}\text { Non-haemorrhagic STEMI } \\
(\mathrm{n}=21)\end{array}$ & p Value \\
\hline LV end diastolic volume index $\left(\mathrm{ml} / \mathrm{m}^{2}\right)$ & $81.0 \pm 21.4$ & $67.1 \pm 11.4$ & $<0.001$ \\
\hline LV end systolic volume index $\left(\mathrm{ml} / \mathrm{m}^{2}\right)$ & $41.1 \pm 17.1$ & $28.3 \pm 7.9$ & $<0.001$ \\
\hline LV ejection fraction $(\%)$ & $51 \pm 11$ & $59 \pm 7$ & $<0.01$ \\
\hline LV mass index $\left(\mathrm{g} / \mathrm{m}^{2}\right)$ & $84 \pm 16$ & $73 \pm 11$ & $<0.01$ \\
\hline Infarct fractional wall thickening (\%) & $4.5 \pm 13.5$ & $23.2 \pm 19.2$ & $<0.0001$ \\
\hline Infarct absolute wall thickening (mm) & $0.4 \pm 1.1$ & $1.6 \pm 1.0$ & $<0.0001$ \\
\hline Infarct end diastolic wall thickness $(\mathrm{mm})$ & $8.5 \pm 2.3$ & $7.8 \pm 1.7$ & NS \\
\hline Infarct (\% LV) & $23.8 \pm 8.2$ & $12.0 \pm 5.9$ & $<0.0001$ \\
\hline T2 oedema (\% LV) & $37.3 \pm 12.5$ & $30.2 \pm 12.5$ & $<0.05$ \\
\hline Early MVO (\% LV) & $19.0 \pm 11.0$ & $0.1 \pm 0.7$ & $<0.0001$ \\
\hline Late MVO (\% LV) & $6.6 \pm 4.8$ & Not detected & $<0.0001$ \\
\hline
\end{tabular}

Values given as mean $\pm S D$.

LV, left ventricular; MVO, microvascular obstruction; STEMI, ST elevation myocardial infarction. 


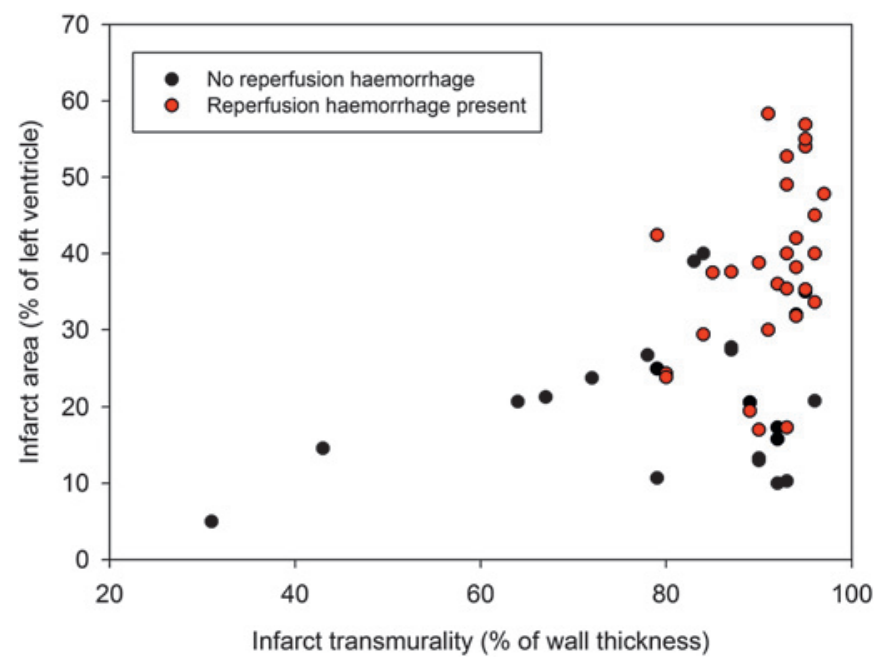

Figure 3 Scatterplot of infarct transmurality and cross-sectional infarct area. Severe reperfusion injury (red points) is more prevalent in larger infarcts where necrosis is almost transmural.

the susceptibility effects of subacute haemorrhage than spin echo imaging and does not rely on the presence of myocardial oedema. ${ }^{30}$ Further research will be needed to determine the independent predictive contribution of reperfusion haemorrhage in ventricular function and remodelling. We identified infarct volume as the only independent predictor of baseline ejection fraction, and this reflected the strong colinearity of two independent variables (haemorrhage and MVO) in the regression model. As well as risk stratification, T2*-CMR may also prove useful in the investigation of cardioprotective therapies by providing a specific biomarker of reperfusion-induced tissue injury.

A limitation of this study was that no histopathological correlation with imaging findings was possible as all of the patients survived, and the validity of our observations is based on published animal and ex vivo studies. The sizing of haemorrhage was based on a $\mathrm{T}^{*}$ threshold derived from comparison with remote myocardium; however, the maturation of blood products in the first week following PCI may affect the

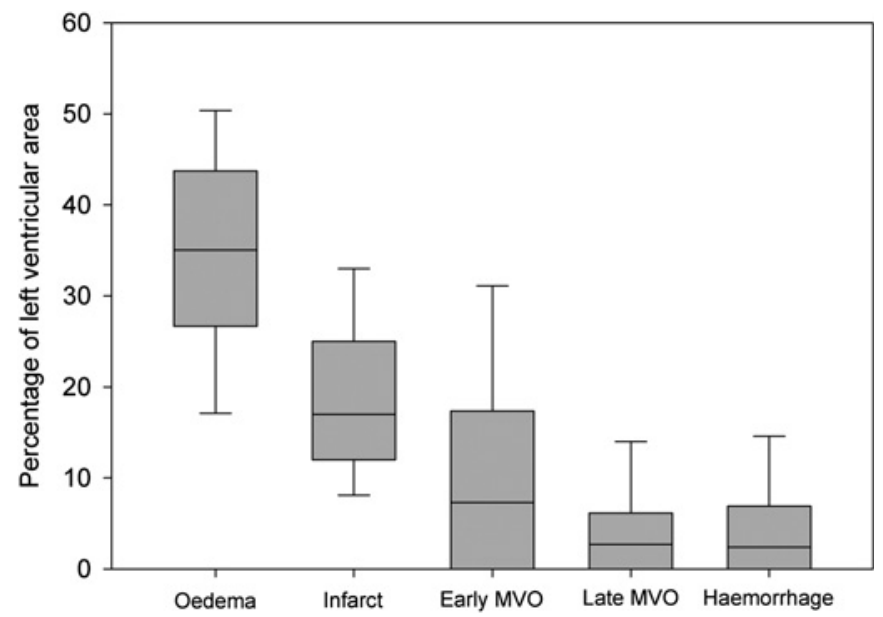

Figure 4 Box plot of the relative areas of ischaemia-reperfusion injury. Characteristics of the areas of infarct tissue determined with cardiac MRI measuring oedema with T2-SPIR, early microvascular obstruction (MVO) with early enhancement, late MVO and infarct size with delayed enhancement, and haemorrhage with $\mathrm{T}^{*}$ mapping (boxes show the median value and are bounded by the 25th and 75th centiles, error bars are at the 10th and 90th centiles). degree of paramagnetic susceptibility. T2* imaging was also only performed at one level in the heart. In common with previous studies, we observed that the extent of apparent MVO decreases with time following gadolinium administration. ${ }^{23}$ Our findings demonstrated a stronger correlation between haemorrhage and the extent of MVO on delayed sequences, which suggests that the haemorrhagic infarct core has the most severe impairment of microvascular flow. However, the optimal methods for sizing MVO have not been determined.

\section{CONCLUSION}

Severe reperfusion injury may occur when there is near transmural myocardial necrosis despite early and successful revascularisation. Reperfusion haemorrhage is closely associated with the development of MVO. These findings indicate that, once advanced necrosis has developed, the potential for severe myocardial reperfusion injury is significantly enhanced.

Funding Medical Research Council, UK.

Competing interests None.

Ethics approval This study was conducted with the approval of the Hammersmith Hospital research ethics committee.

Provenance and peer review Not commissioned; externally peer reviewed.

\section{REFERENCES}

1. Nordmann AJ, Hengstler P, Harr $T$, et al. Clinical outcomes of primary stenting versus balloon angioplasty in patients with myocardial infarction: a meta-analysis of randomized controlled trials. Am J Med 2004;116:253-62.

2. Prasad A, Stone GW, Holmes DR, et al. Reperfusion injury, microvascular dysfunction, and cardioprotection: the "dark side" of reperfusion. Circulation 2009:120:2105-12.

3. Yellon DM, Hausenloy DJ. Myocardial reperfusion injury. N Engl J Med 2007:357:1121-35

4. Piper HM, Garcia-Dorado D, Ovize M. A fresh look at reperfusion injury. Cardiovaso Res 1998;38:291-300.

5. Pennell D. Myocardial salvage: retrospection, resolution, and radio waves. Circulation 2006:113:1821-3.

6. Carlsson M, Ubachs JFA, Hedstrom E, et al. Myocardium at risk after acute infarction in humans on cardiac magnetic resonance: quantitative assessment during follow-up and validation with single-photon emission computed tomography. JACC Cardiovasc Imaging 2009;2:569-76.

7. Aletras $\mathbf{A H}$, Tilak GS, Natanzon A, et al. Retrospective determination of the area at risk for reperfused acute myocardial infarction with T2-weighted cardiac magnetic resonance imaging: histopathological and displacement encoding with stimulated echoes (DENSE) functional validations. Circulation 2006;113:1865-70.

8. Tilak GS, Hsu L-Y, Hoyt RF, et al. In vivo T2-weighted magnetic resonance imaging can accurately determine the ischemic area at risk for 2-day-old nonreperfused myocardial infarction. Invest Radiol 2008;43:7-15.

9. Higginson LA, White F, Heggtveit HA, et al. Determinants of myocardial hemorrhage after coronary reperfusion in the anesthetized dog. Circulation 1982:65:62-9.

10. Kloner RA, Ellis SG, Lange R, et al. Studies of experimental coronary artery reperfusion. Effects on infarct size, myocardial function, biochemistry, ultrastructure and microvascular damage. Circulation 1983:68:8-15.

11. Lotan CS, Bouchard A, Cranney GB, et al. Assessment of postreperfusion myocardial hemorrhage using proton NMR imaging at 1.5 T. Circulation 1992;86:1018-25.

12. Rochitte CE, Lima JA, Bluemke DA, et al. Magnitude and time course of microvascular obstruction and tissue injury after acute myocardial infarction. Circulation 1998;98:1006-14.

13. O'Regan DP, Ahmed R, Karunanithy N, et al. Reperfusion hemorrhage following acute myocardial infarction: assessment with $\mathrm{T} 2{ }^{*}$ mapping and effect on measuring the area at risk. Radiology 2009;250:916-22.

14. O'Regan DP, Ahmed R, Neuwirth C, et al. Cardiac MRI of myocardial salvage at the peri-infarct border zones after primary coronary intervention. Am J Physiol Heart Circ Physiol 2009;297:340-6.

15. Heiberg $\mathbf{E}$, Ugander $\mathrm{M}$, Engblom $\mathrm{H}$, et al. Automated quantification of myocardial infarction from MR images by accounting for partial volume effects: animal, phantom, and human study. Radiology 2008:246:581-8

16. Ganame J, Messalli G, Dymarkowski S, et al. Impact of myocardial haemorrhage on left ventricular function and remodelling in patients with reperfused acute myocardial infarction. Eur Heart J 2009:30:1440-9.

17. Reimer KA, Jennings RB. The "wavefront phenomenon" of myocardial ischemic cell death. II. Transmural progression of necrosis within the framework of ischemic bed size (myocardium at risk) and collateral flow. Lab Invest 1979;40:633-44.

18. van den Bos EJ, Baks T, Moelker AD, et al. Magnetic resonance imaging of haemorrhage within reperfused myocardial infarcts: possible interference with iron oxide-labelled cell tracking? Eur Heart J 2006;27:1620-6. 
19. Basso C, Corbetti F, Silva C, et al. Morphologic validation of reperfused hemorrhagic myocardial infarction by cardiovascular magnetic resonance. Am J Cardiol 2007; 100:1322-7

20. Busk M, Kaltoft A, Nielsen SS, et al. Infarct size and myocardial salvage after primary angioplasty in patients presenting with symptoms for $<12 \mathrm{~h}$ vs. $12-72 \mathrm{~h}$. Eur Heart J 2009:30:1322-30.

21. Fishbein MC, Y-Rit J, Lando U, et al. The relationship of vascular injury and myocardial hemorrhage to necrosis after reperfusion. Circulation 1980;62:1274-9.

22. Bekkers SCAM, Yazdani SK, Virmani R, et al. Microvascular obstruction: underlying pathophysiology and clinical diagnosis. J Am Coll Cardiol 2010;55:1649-60.

23. Rochitte CE. Microvascular obstruction: the final frontier for a complete myocardial reperfusion. J Am Coll Cardiol 2008;51:2239-40.

24. Wu KC, Zerhouni EA, Judd RM, et al. Prognostic significance of microvascular obstruction by magnetic resonance imaging in patients with acute myocardia infarction. Circulation 1998:97:765-72.
25. Kim CB, Braunwald E. Potential benefits of late reperfusion of infarcted myocardium. The open artery hypothesis. Circulation 1993;88:2426-36.

26. Marroquin 0C, Lamas GA. Beneficial effects of an open artery on left ventricular remodeling after myocardial infarction. Prog Cardiovasc Dis 2000:42:471-83.

27. Dzavik V, Buller CE, Lamas GA, et al. Randomized trial of percutaneous coronary intervention for subacute infarct-related coronary artery occlusion to achieve longterm patency and improve ventricular function: the Total Occlusion Study of Canada (TOSCA)-2 trial. Circulation 2006;114:2449-57.

28. Hochman JS, Lamas GA, Buller CE, et al. Coronary intervention for persistent occlusion after myocardial infarction. N Engl J Med 2006;355:2395-407.

29. Beek AM, Nijveldt R, van Rossum AC. Intramyocardial hemorrhage and microvascular obstruction after primary percutaneous coronary intervention. Int J Cardiovasc Imaging 2010;26:49-55.

30. Bradley WG. MR appearance of hemorrhage in the brain. Radiology 1993:189:15-26. 